\title{
Resonant magnetic field control of elastic scattering in cold ${ }^{85} \mathrm{Rb}$
}

\author{
J. L. Roberts, N. R. Claussen, James P. Burke, Jr., Chris H. Greene, E. A. Cornell,* and C. E. Wieman \\ JILA and the Department of Physics, University of Colorado, Boulder, CO
}

80309-0440

(October 24, 2018)

\begin{abstract}
A magnetic field dependent Feshbach resonance has been observed in the elastic scattering collision rate between atoms in the $F=2, M=-2$ state of ${ }^{85} \mathrm{Rb}$. Changing the magnetic field by several Gauss caused the collision rate to vary by a factor of $10^{4}$, and the sign of the scattering length could be reversed. The resonance peak is at 155.2(4) $\mathrm{G}$ and its width is 11.6(5) G. From these results we extract much improved values for the three quantities that characterize the interaction potential: the van der Waals coefficient $\mathrm{C}_{6}$, the singlet scattering length $a_{S}$, and the triplet scattering length $a_{T}$.
\end{abstract}

Very low-temperature collision phenomena can be quite sensitive to applied electromagnetic fields. Several groups have altered inelastic collision rates in optical traps by applying laser fields [1]. There have also been numerous proposals [2 6] for using laser and static electric or magnetic fields to influence the S-wave scattering length $(a)$, and equivalently, the elastic collision cross sections $\left(\sigma=8 \pi a^{2}\right)$ between cold atoms. Particularly notable is the prediction by Verhaar and co-workers [2] that as a function of magnetic field there should be Feshbach resonances in collisions between cold $(\sim \mu \mathrm{K})$ alkali atoms. These resonances were predicted to cause dramatic changes in the cross section, and to even allow the sign of the scattering length to be changed. Such resonances are very interesting collision physics, but they also offer a means to manipulate Bose-Einstein condensates (BEC), the properties of which are primarily determined by the scattering length. Some time ago we searched for such resonances in ${ }^{133} \mathrm{Cs}$ and ${ }^{87} \mathrm{Rb}$, without success [7,8]. This was not surprising because there was enormous uncertainty as to the positions and widths of the predicted resonances. However, recent photoassociation spectroscopy has greatly improved the knowledge of the alkali interatomic interaction potentials, allowing these resonance parameters to be predicted with much less uncertainty [9]. As we show below, the fact that the widths and positions of these resonances are so sensitive to the interatomic potentials makes their measurement a good way to determine these potentials.

The improved predictions for the resonance positions facilitated their observation. In the past few months Feshbach resonances have been observed in both ${ }^{23} \mathrm{Na} 10$ and ${ }^{85} \mathrm{Rb} 11$. In the sodium work, two magnetic field dependent processes were observed: 1) a change in the expansion rate of BEC due to a change in the scattering length, and 2) an enormous, and as yet unexplained, increase in the loss rate. This loss rate precluded the study of collisions in the interesting field regime near the center of the resonance where the scattering length changes sign; the width of the resonance was not measured. In the rubidium work 11] the resonance was detected as a magnetic field dependence of the photoassociation spectra. The resulting resonance width was measured to be far larger than originally predicted.

Here we report the study of a Feshbach resonance in the elastic collision cross section between atoms in the $F=2, M=-2$ state of ${ }^{85} \mathrm{Rb}$. By changing the DC magnetic field we are able to change the collision rate by 4 orders of magnitude, and explore regions of positive, negative, and essentially zero scattering length. We determine the width and position of the resonance about a factor of ten more precisely than in Ref. [11, and from this data we improve the accuracy of the $\mathrm{Rb}$ interaction potential substantially. In contrast to Ref. [10, we do not observe two or three body loss at resonance because we work at much lower densities $\left(10^{9}\right.$ atoms $\left./ \mathrm{cm}^{3}\right)$.

We measured the collision rates using the technique of "cross dimensional mixing," as in our earlier work [7]. In this technique, a nonisotropic distribution of energy is created in a magnetically trapped cloud of atoms, and the time for the cloud to reequilibrate by elastic collisions is measured. The apparatus used is identical to that used in our previous work on BEC in ${ }^{87} \mathrm{Rb}[12$. It is a double magneto-optic trap (MOT) system in which multiple samples of atoms are trapped in a relatively high-pressure chamber, and then transferred to a second MOT in a lowpressure chamber. The second MOT is then turned off and a "baseball coil" magnetic trap is turned on around them. The atoms are then cooled by forced evaporation.

After the atoms are evaporatively cooled to the desired temperature, the energy in the radial direction (and correspondingly the square of the width of the trapped cloud) is reduced to about 0.6 that of the axial direction. This is done by decreasing the frequency of the RF "knife" used for the evaporative cooling more rapidly than the cloud can equilibrate. The bias magnetic field is then adiabatically ramped to the selected value, and the cloud is allowed to equilibrate for a fixed time. The shape of the cloud is then measured by absorptive imaging. This is repeated for different equilibration times, and the aspect ratios of the cloud vs. time are fit to an exponential to determine the equilibration time con- 
stant $\tau_{e q}$. As discussed in Ref. [13], $\tau_{e q}$ is proportional to the inverse of the elastic collision rate 14 . From the measured trap oscillation frequencies and the measured shape and optical depth, we determine the temperature $T$ and average density $\langle n\rangle$ of the cloud. The magnetic field value at the center of the cloud is determined to $\pm 0.1 \mathrm{G}$ by finding the RF frequency that resonantly drives spin flip transitions for the atoms at that position and using the Breit-Rabi equation. The spread in magnetic field across the cloud scales as $T^{1 / 2}$, and is $0.6 \mathrm{G}$ FWHM for a $0.5 \mu \mathrm{K}$ cloud.

The measured equilibration times depend on $B$ and $T$ and vary from $0.15 \mathrm{~s}$ to nearly $2000 \mathrm{~s}$. To display data taken at several values of both density and temperature, we convert it to the normalized equilibration rate, $\Gamma_{n o r m}=1 /\langle n v\rangle \tau_{e q}$. Figure 1 displays the data versus mean magnetic field. $\Gamma_{\text {norm }}$ has units of cross-section and in fact is proportional to an empirical average over the field- and energy-dependent elastic scattering crosssection $\sigma(B, E)$. To calculate this average is problematic - a detailed Monte Carlo simulation of particle trajectories 15] is probably required. However in the lowtemperature limit it is clear that the maximum and minimum values of $\Gamma_{\text {norm }}$ will occur at the same magnetic field as the peak and zero of $\sigma(B, E=0)$. For a rough qualitative comparison, in Fig. 1 we have also plotted the theoretically predicted values of $\sigma(B, E)$ for two temperatures. These predictions are calculated using the atomic potentials determined from the observed maximum and minimum values of the relaxation rate, as discussed below. Both the cross section curves and the equilibration data show the same qualitative features. For temperatures below a few $\mu \mathrm{K}$, there is a slightly asymmetric peak near $155 \mathrm{G}$, and the width and height of this peak are strong functions of temperature. At $167 \mathrm{G}$ there is a profound drop in the rate. This dip is quite asymmetric, but the shape is relatively insensitive to $T$, and at the bottom (field value $B_{\text {min }}$ ) the rate is essentially zero. The field value of the peak ( $\left.B_{\text {peak }}\right)$ is customarily defined to be the position of the Feshbach resonance, and the resonance width, $\Delta$ is the distance between $B_{\text {peak }}$ and $B_{\text {min }}$ [11. The scattering length is positive for field values between $B_{\text {peak }}$ and $B_{\text {min }}$, and is negative for field values below $B_{\text {peak }}$ or above $B_{\text {min }}$. This dependence of the sign is expected from previous theory. The observation that $B_{\text {peak }}$ is at a lower field than $B_{\text {min }}$ provides experimental confirmation that the scattering length is negative away from the resonance.

Because the functional form of the normalized equilibration rate is not known, the desired quantities, $B_{\text {peak }}$ and $B_{\min }$ cannot be found by a detailed fit to all of the data. We determine them by fitting a simple smooth curve to only the few highest (or lowest) points at each temperature below $5 \mu \mathrm{K}$, and assigning correspondingly conservative error bars that more than span the values determined for all temperatures [16]. We find $B_{\text {peak }}=$
$155.2(4) \mathrm{G}$ and $B_{\min }=166.8(3) \mathrm{G}$, giving a resonance width $\Delta=11.6$ (5) G. The values for $B_{\text {peak }}$ and $\Delta$ are reasonably consistent with the less precise values $164(7)$ and 8.4 (3.7) G measured in Ref. [11]. In our experiment, the accuracy of the peak position is much higher primarily because of better field calibration. The better accuracy in the width is largely because the photoassociation measurement is a somewhat less direct way to observe the resonance, and so substantial and somewhat uncertain corrections are required to go from observed signal widths to the actual resonance width.

We can now use these measured quantities to determine the singlet and triplet Born-Oppenheimer potentials. The accuracy of predicted cold collision observables hinges on the quality of these potentials used in the radial Schrödinger equation for the nuclear motion. Accurate determination of these potentials in turn relies primarily on three parameters, the long range van der Waals coefficient $\mathrm{C}_{6}$ and the zero-energy singlet and triplet phases. The phases are usually tabulated in terms of singlet $a_{S}$ and triplet $a_{T}$ scattering lengths calculated with hyperfine terms omitted from the radial equation.

The Feshbach resonance involves states with both singlet and triplet character; consequently the present determination of resonance width and position versus $B$ can be translated with the aid of theory into greatly improved values for the singlet and triplet scattering lengths. The old "nominal" values of the scattering lengths and $\mathrm{C}_{6}$ (optimized to achieve agreement with previous measurements) predicted the position of this Feshbach resonance reasonably well, but, as in Ref. 11] the predicted width was much smaller than observed. This width reflects the coupling of bound and continuum channels, and is primarily controlled by the difference between the singlet and triplet scattering lengths. It is inconvenient to tabulate results as a function of singlet and triplet scattering lengths for ${ }^{85} \mathrm{Rb}$, because both are very close to lying on top of a divergent pole. Accordingly, we present the results of our analysis in terms of a dissociation phase $\nu_{\mathrm{D}}$ as in Ref. [11]. We define the corresponding singlet $\nu_{\mathrm{DS}}$ or triplet $\nu_{\mathrm{DT}}$ dissociation phase in terms of the scattering length through the relation [17]: $\nu_{\mathrm{D}}=(1 / \pi) \cot ^{-1}\left(a / a_{\text {ref }}-1\right)$ where $a_{\text {ref }}=\Gamma(5 / 4) /[\sqrt{8} \Gamma(3 / 4)]\left(2 m \mathrm{C}_{6} / \hbar^{2}\right)^{1 / 4}$ 18 and $m$ is the reduced mass of the atomic pair. The dissociation phase is related to the short range quantum defect $\mu^{\mathrm{sr}}$ presented in Ref. [19] by $\nu_{\mathrm{D}} \cong \mu^{\mathrm{sr}}-1 / 4$. The quantum defect method developed in Ref. [19] was used to generate the theory curves in Fig. 1.

We have adjusted the singlet and triplet Rb-Rb potential curves at small distances until the theoretical calculation agrees simultaneously with the present measurements of $B_{\text {peak }}$ and $\Delta$, to within the experimental uncertainties. For a given $\mathrm{C}_{6}$, this severely constrains the values of both $\nu_{\mathrm{DS}}$ and $\nu_{\mathrm{DT}}$ (see Fig. 2). However, the position and width are not sufficient to totally constrain 
all three parameters $\mathrm{C}_{6}, \nu_{\mathrm{DS}}$, and $\nu_{\mathrm{DT}}$. In order to obtain values for all the parameters, we also require that our triplet potentials predict the $g$-wave shape resonance in an energy range consistent with the measured value given in Ref. 20.

The results of this analysis are presented in Fig. 2. It is immediately clear from the figure that accurate measurement of Feshbach resonances are an extremely precise method for determining threshold properties of the interatomic potentials. In fact, our measurement has allowed us to reduce the combined $\mathrm{C}_{6}, \nu_{\mathrm{DS}}$, and $\nu_{\mathrm{DT}}$ parameter space by roughly a factor of 80 . As discussed in Ref. [11, the position of the resonance depends mostly on $\mathrm{C}_{6}$ and the sum $\nu_{\mathrm{DS}}+\nu_{\mathrm{DT}}$. The width depends mostly on the difference $\nu_{\mathrm{DS}}-\nu_{\mathrm{DT}}$. As Fig. 2 shows, the allowed $\nu_{\mathrm{DS}}$, $\nu_{\mathrm{DT}}$ parameter region is an extremely correlated function of $\mathrm{C}_{6}$. In particular, we find that the nominal value of the sum and difference coordinates are linear functions of $\mathrm{C}_{6}$ and the uncertainties in the coordinates are constant. Accordingly, we can represent the allowed parameter region in the following manner:

$\nu_{\mathrm{DS}}+\nu_{\mathrm{DT}}=-0.0442+2.2\left(10^{-4}\right)\left(\mathrm{C}_{6}-\overline{\mathrm{C}}_{6}\right) \pm 0.0005$ and $\nu_{\mathrm{DS}}-\nu_{\mathrm{DT}}=0.0666-6.2\left(10^{-5}\right)\left(\mathrm{C}_{6}-\overline{\mathrm{C}}_{6}\right) \pm 0.003$ where $\overline{\mathrm{C}}_{6}=4700$. Converting the dissociation phases into scattering lengths, we find reasonable agreement with previous work 21 23. We find for the nominal $\mathrm{C}_{6}$ the values (in a.u.) $\mathrm{a}_{t}\left({ }^{85} \mathrm{Rb}\right)=-363 \pm 10, \mathrm{a}_{s}\left({ }^{85} \mathrm{Rb}\right)=2300_{-200}^{+300}$, $\mathrm{a}_{t}\left({ }^{87} \mathrm{Rb}\right)=109.3 \pm 0.4$, and $\mathrm{a}_{s}\left({ }^{87} \mathrm{Rb}\right)=92.7 \pm 0.4$. Our value for $\mathrm{C}_{6}, 4700(50)$ a.u. is slightly higher (and with smaller uncertainty) than a previous analysis based solely on the $g$-wave shape resonance 20]. We have confirmed that several scattering observables predicted by the new Born-Oppenheimer potentials are consistent with previous measurements. Specifically, the new potentials predict a broad $d$-wave shape resonance 25 in ${ }^{87} \mathrm{Rb}$, the scattering length ratio $a_{2,1} / a_{1,-1}$ [26] and the thermallyaveraged $|2,2\rangle+|1,-1\rangle$ inelastic rate constant 27] that are consistent with previous measurements. We also find 10 of the 12 measured $d$-wave bound states [22] within the $2 \sigma$ error bars 24 . The new potentials also permit us to predict additional ${ }^{85} \mathrm{Rb}$ Feshbach resonances [6], at $B_{\text {peak }}=226.5 \pm 4.8 \mathrm{G}$ with a width of $0.01 \mathrm{G}$, and at $B_{\text {peak }}=535.8 \pm 4.0 \mathrm{G}$ with a width of $2.2 \pm 0.2 \mathrm{G}$.

We have shown that precise measurements of the Feshbach resonance in the elastic scattering cross section provide unprecedented knowledge of the Born-Oppenheimer potentials that control Rb-Rb scattering processes at sub-mK temperatures. We have also used this resonance to enhance evaporative cooling of ${ }^{85} \mathrm{Rb}$ in a low density magnetic trap, and change the scattering length from negative to positive. These capabilities should allow novel studies of BEC in the future.

We are pleased to acknowledge valuable conversations with Dan Heinzen, Boudwijn Verhaar, and John Bohn, as well as the assistance of Richard Ghrist and Eric Burt in the early stages of the work. NSF, ONR, and NIST supported this work.

*Quantum Physics Division, National Institute of Standards and Technology

[1] V. Sanchez-Villicana, S. D. Gensemer, and P. L. Gould, Phys. Rev. A 54, R3730 (1996); and references therein.

[2] E. Tiesinga, A. J. Moerdijk, B. J. Verhaar, and H. Stoof, Phys. Rev. A 46, R1167 (1992); E. Tiesinga, B. J. Verhaar, and H. Stoof, Phys. Rev. A 47, 4114 (1993).

[3] M. Marinescu and L. You (submitted to PRL).

[4] P. O. Fedichev et al., Phys. Rev. Lett. 77, 2913 (1996).

[5] J. L. Bohn and P. S. Julienne, Phys. Rev. A 56, 1486 (1997).

[6] A. J. Moerdijk et al., Phys. Rev. A 51, 4852 (1995); J. M. Vogels et al., Phys. Rev. A 56, R1067 (1997).

[7] C. R. Monroe, E. A. Cornell, C. A. Sackett, C. J. Myatt, and C. E. Wieman, Phys. Rev. Lett. 70, 414 (1993).

[8] N. R. Newbury, C. J. Myatt, and C. E. Wieman, Phys. Rev. A 51, R2680 (1995).

[9] For a recent review on this subject, see for instance: P. S. Julienne, J. Res. Natl. Inst. Stand. Technol. 101, 487 (1996).

[10] S. Inouye, M. R. Andrews, J. Stenger, H. J. Miesner, D. M. Stamper-Kurn, and W. Ketterle, Nature 392, 151 (1998).

[11] Ph. Courteille, R. S. Freeland, and D. J. Heinzen, F. A. van Abeelen, and B. J. Verhaar, Phys. Rev. Lett. 81, 69 (1998).

[12] C. J. Myatt, N. R. Newbury, R. W. Ghrist, S. Loutzenhiser, and C. E. Wieman, Opt. Lett. 21, 290 (1996).

[13] C. R. Monroe, 1992 Doctoral Dissertation in Physics, University of Colorado.

[14] The proportionality constant is about 2.6 when the elastic cross section is temperature-independent, which is decidedly not the case here.

[15] D. Gury-Odelin, J. Sding, P. Desbiolles, and J. Dalibard, Optics Express 2, 323 (1998).

[16] The three values of $B_{\text {peak }}$ determined for the $0.5,1$, and $0.75 \mu \mathrm{K}$ data (the latter not shown in Fig. 1 to avoid cluttering it excessively) can be determined to about the same precision and agree to within $0.4 \mathrm{G}$. The value of $B_{\min }$ is primarily based on the $3 \mu \mathrm{K}$ data.

[17] F. H. Mies, C. J. Williams, P. S. Julienne, and M. Krauss, J. Res. Natl. Inst. Stand. Technol. 101, 521 (1996).

[18] G. F. Gribakin and V. V. Flambaum, Phys. Rev. A 48, 546 (1993).

[19] J. P. Burke, Jr., C. H. Greene, and J. L. Bohn, (submitted to PRL, June 1998). The scattering length is given in terms of the quantum defect by: $a=-C^{2} \tan \left(\pi \mu^{\mathrm{sr}}\right) /[1+$ $\left.\mathcal{G}(0) \tan \left(\pi \mu^{\mathrm{sr}}\right)\right]$, where $C^{2}=0.957217\left(2 m \mathrm{C}_{6} / \hbar^{2}\right)^{1 / 4}$ and $\mathcal{G}(0)=-1.00260$.

[20] H.M.J.M. Boesten, C. C. Tsai, B. J. Verhaar, and D. J. Heinzen, Phys. Rev. Lett. 77, 5194 (1996).

[21] J. P. Burke, Jr., J. L. Bohn, B. D. Esry, and C. H. Greene, Phys. Rev. Lett. 80, 2097 (1998). 
[22] C. C. Tsai, R. S. Freeland, J. M. Vogels, H.M.J.M. Boesten, B. J. Verhaar, and D. J. Heinzen, Phys. Rev. Lett. 79, 1245 (1997).

[23] P. S. Julienne, F. H. Mies, E. Tiesinga, and C. J. Williams, Phys. Rev. Lett. 78, 1880 (1997); J. P. Burke, Jr., J. L. Bohn, B. D. Esry, and C. H. Greene, Phys. Rev. A 55, R2511 (1997).

[24] Those in disagreement are likely due to somewhat overly optimistic error bars for the positions of the bound states as determined by photoassociation experiments. (Dan Heinzen, private communication).

[25] H.M.J.M. Boesten, C. C. Tsai, J. R. Gardner, D. J. Heinzen, and B. J. Verhaar, Phys. Rev. A 55636 (1997).

[26] M. R. Matthews, D. S. Hall, D. S. Jin, J. R. Ensher, C. E. Wieman, E. A. Cornell, F. Dalfovo, C. Minniti, and S. Stringari, Phys. Rev. Lett. 81, 243 (1998).

[27] C. J. Myatt, E. A. Burt, R. W. Ghrist, E. A. Cornell, and C. E. Wieman, Phys. Rev. Lett. 78, 586 (1997).

FIG. 1. (a) The data points show the measured equilibration rate divided by average density and velocity $\left(\Gamma_{n o r m}=1 /\langle n v\rangle \tau_{e q}\right)$ vs. magnetic field $B$. Data are shown for clouds at five different temperatures: $0.3 \mu \mathrm{K}$ (filled triangles), $0.5 \mu \mathrm{K}(\bullet), 1.0 \mu \mathrm{K}(\circ), 3.5 \mu \mathrm{K}(\nabla)$, and $9.0 \mu \mathrm{K}$ (filled squares). The lines are calculations for the thermally averaged elastic cross sections (not equilibration rates!) and hence are not expected to fit the data points. The solid line corresponds to $0.5 \mu \mathrm{K}$ and the dot-dashed to $9.0 \mu \mathrm{K}$. (b),(c) Expanded views of the regions of maximum and minimum cross section.

FIG. 2. Comparison of the allowed $\nu_{\mathrm{DS}}, \nu_{\mathrm{DT}}$ parameter space based on this work and previous measurements for a constant value of $\mathrm{C}_{6}$. The rectangle is the allowed range from Ref. [22]. The large diamond is the range from Ref. [11]. The small filled diamond is the range from this work. Note that the rectangle and large diamond constraints were established using a different value of $\mathrm{C}_{6}$ from the one used in this work (4550 instead of 4700 a.u.). As is discussed in the text, the position of the constrained regions depends linearly upon $\mathrm{C}_{6}$, which is indicated by the small connected diamonds on either side of the small filled diamond. These diamonds show the effect of 50 a.u. uncertainty in $\mathrm{C}_{6}$. The arrows indicate sensitivity to changes in the resonance width and position in this paramter space. 


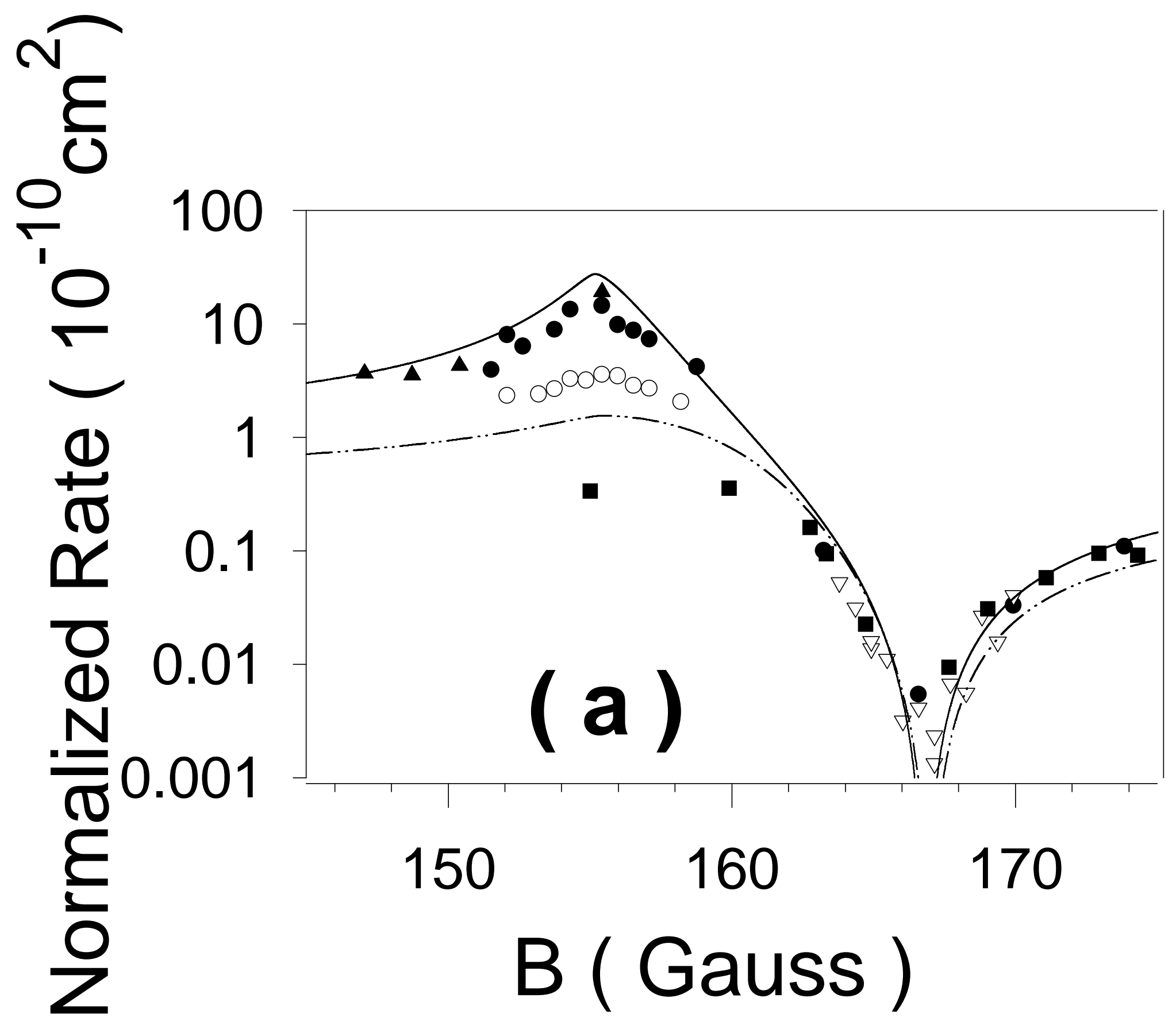




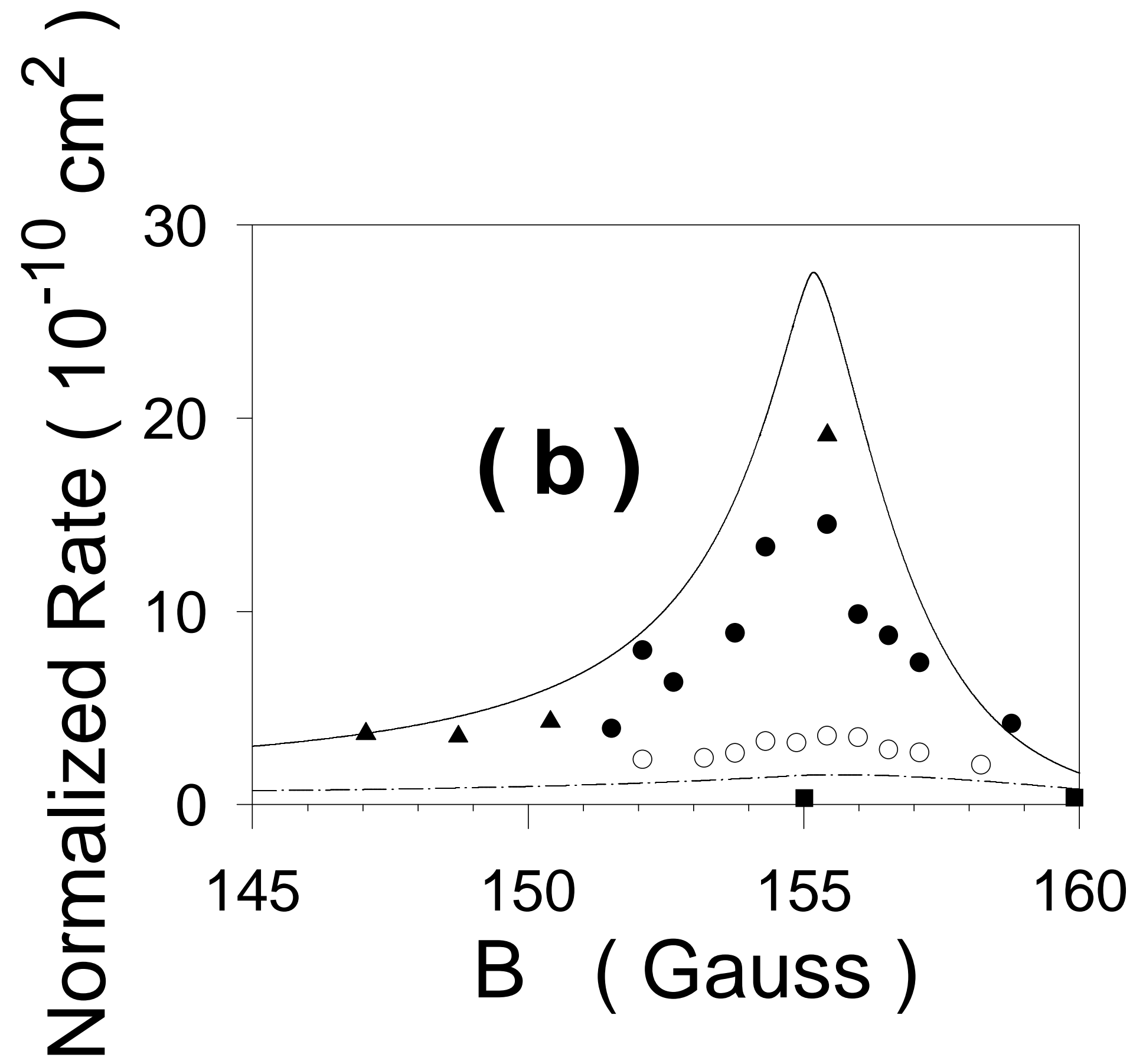


N

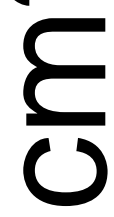

0

T

0.15

I

응

단

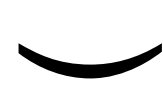

(1)

సั

ฯ

ర

$\Phi$

N $\quad 0.00$

ত্

0.10

0.05

0.00

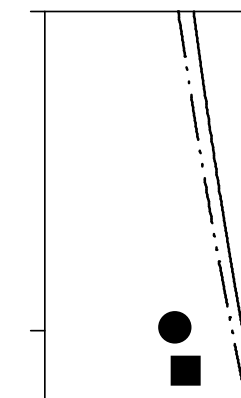

$\frac{E}{2}$

B (Gauss )

170

175
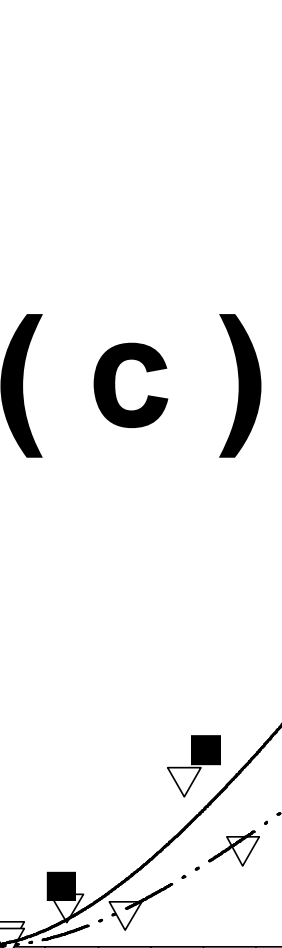

7.

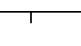




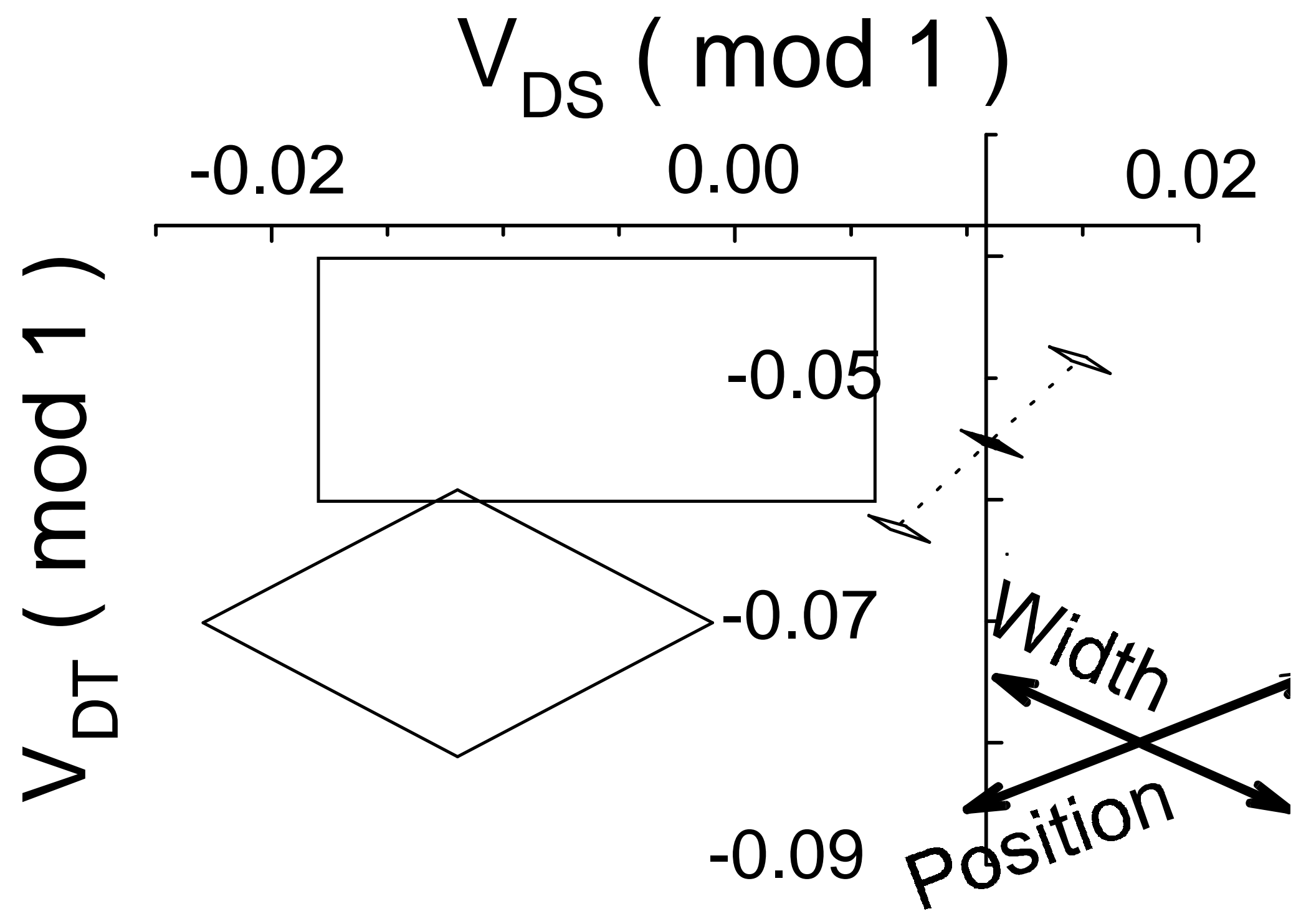

